\title{
Glycoprotein Ib $\alpha$ Kozak polymorphism in patients presenting with early-onset acute coronary syndrome
}

\author{
Ebru Golcuk ${ }^{1}$, Kivanc Yalin ${ }^{1}$, Cansu Selcan Akdeniz², Erhan Teker ${ }^{3}$, Başak Teker ${ }^{4}$, \\ Veysel Sabri Hancer ${ }^{4}$, Ibrahim Altun ${ }^{5}$, Murat Sezer ${ }^{6}$, Reyhan Diz Kucukkaya ${ }^{7}$, Aytac Oncul ${ }^{6}$
}

\author{
${ }^{1}$ Department of Cardiology, Bayrampasa Kolan Hospital, Istanbul, Turkey \\ ${ }^{2}$ Department of Cardiology, Faculty of Medicine, Istanbul Bilim University, Istanbul, \\ Turkey \\ ${ }^{3}$ Cardiology Clinic, Giresun State Hospital, Giresun, Turkey \\ ${ }^{4}$ Department of Molecular Genetics, Faculty of Medicine, Istanbul University, Istanbul, \\ Turkey \\ ${ }^{5}$ Department of Cardiology, Faculty of Medicine, Muğla Sıtkı Kocman University, \\ Mugla, Turkey \\ ${ }^{6}$ Department of Cardiology, Faculty of Medicine, Istanbul University, Istanbul, Turkey \\ ${ }^{7}$ Department of Internal Medicine-Hematology, Faculty of Medicine, Istanbul Bilim \\ University, Istanbul, Turkey
}

Submitted: 1 April 2016

Accepted: 19 July 2016

Arch Med Sci 2018; 14, 4: 788-793

DOI: https://doi.org/10.5114/aoms.2016.63278

Copyright $\odot 2016$ Termedia \& Banach

\section{Abstract}

Introduction: Glycoprotein Ib $(\mathrm{GPI} \alpha)$ receptor is the chief molecule responsible for initial platelet adhesion to the subendothelium. A thymidine to cytosine single nucleotide substitution at position -5 from the ATG start codon characterizes the Kozak sequence polymorphism. The Kozak sequence polymorphism may increase the surface expression of GPIb $\alpha$ and contribute to thrombogenesis. We evaluated the allele frequencies of GPIb $\alpha$ Kozak sequence polymorphism in the Turkish population and examined the relationship between GPIba Kozak sequence polymorphism and early-onset acute coronary syndrome (ACS).

Material and methods: This study enrolled 200 patients (122 male, $78 \mathrm{fe}$ male, mean age: $39 \pm 5$ years) and 200 healthy control subjects (110 male, 90 female, $41 \pm 4$ years). The patient group was composed of patients admitted to our coronary care unit with early-onset ACS and patients who attended to our cardiology outpatient clinic after hospital discharge with a diagnosis of early-onset ACS.

Results: Kozak polymorphism frequencies in patients and control subjects did not differ significantly (23\% versus $22.5 \%, p=0.812$, respectively). In patients who presented with non-ST elevation myocardial infarction (NSTEMI), the frequency of GPIba Kozak polymorphism was borderline significantly higher when compared with patients who presented with ST elevation myocardial infarction (STEMI) (35\% vs. $20 \%, p=0.05$, respectively). Allele frequencies of $\mathrm{T}$ and $\mathrm{C}$ were calculated to be 0.873 and 0.128 .

Conclusions: Although the frequency of GPIba Kozak polymorphism did not differ significantly in early-onset ACS patients versus control subjects, Kozak polymorphism frequency was borderline significantly higher in patients who presented with NSTEMI when compared to patients with STEMI.

Key words: Turkish population, acute coronary syndrome, glycoprotein Ib $\alpha$ Kozak polymorphism.

\author{
Corresponding author: \\ Ebru Golcuk \\ Department of Cardiology \\ Bayrampasa Kolan Hospital \\ Terazidere Mahallesi \\ 60. Yıl Caddesi No: 3 \\ Bayrampasa \\ 34144 Istanbul, Turkey \\ Phone: +90 2125390539 \\ Fax: +902125628484 \\ E-mail: ebru_glck@yahoo. \\ co.nz
}




\section{Introduction}

Prior studies have documented the hereditability of coronary artery disease (CAD) and its most acute manifestation, myocardial infarction (MI). Development of CAD and $\mathrm{MI}$ is determined by several genes. Many genetic factors have been found to have a role in the pathogenesis of CAD and MI. Many single-nucleotide polymorphisms have been associated with CAD and MI [1-3]. Platelets play a pivotal role in acute coronary syndromes (ACS) and acute myocardial infarction (AMI) [4]. The platelet membrane glycoproteins (GPs) act as receptors for many adhesive proteins, such as collagen, von Willebrand factor (vWF), fibronectin, adenosine diphosphate (ADP) and thrombin. The GPIb/IX/V complex is the major platelet surface receptor for vWF. The platelet receptor GPIb/IX/V is a complex of four membrane-spanning polypeptides - GPIb $\alpha$, GPIb $\beta$, GPIX and GPV - that are synthesized from different genes. GPIb also serves as a thrombin receptor. GPIb $\alpha$ is the largest component of the GPIb/IX/V complex, which mediates the initial adhesion of platelets to the blood vessel wall following injury. A thymidine to cytosine single nucleotide substitution at position -5 from the ATG start codon characterizes the Kozak sequence polymorphism, which is one of the polymorphisms of this receptor. The Kozak polymorphism may increase surface expression of GPIb $\alpha$ and contribute to thrombogenesis.

Recent studies revealed the role of the GPIb $\alpha$ Kozak polymorphism in ACS, ischemic stroke and restenosis after coronary percutaneous interventions [5-11], but there are also some studies in the literature in which this relation could not be demonstrated [12-16].

These results prompted us to examine the presence of Kozak sequence polymorphism of GPIb $\alpha$ in the Turkish population and to determine whether the $C$ allele at this polymorphism has a role in patients presenting with ACS at and under the age of 45 .

\section{Material and methods}

The study was approved by the university ethical committee. Written informed consent was obtained from all patients.

The patient group $(n=200 ; M / F=122 / 78$, aged $39 \pm 5$ years) was composed of patients admitted to the Coronary Care Unit with ACS at and under the age of 45 and patients who attended to our cardiology out-patient clinic after hospital discharge with a diagnosis of ACS at and under the age of 45 .

The diagnosis of ACS was based on typical electrocardiographic and enzymatic criteria and confirmed by echocardiography and coronary an- giography, according to ESC guidelines [17]. The healthy control group ( $n=200 ; M / F=110 / 90$, aged $41 \pm 4$ years) was recruited among students or staff personnel who were checked and judged to be in good health on the basis of their clinical history and on blood tests. Hypertension, diabetes mellitus, hyperlipidemia, family history of ischemic heart disease in at least one first degree relative and smoking were present in 14 (7\%), 4 (2\%), 9 (4.5\%), 29 (14.5\%) and 48 (24\%) of 200 controls, respectively. Forty-nine out of the 200 patients had a diagnosis of non-ST-elevation myocardial infarction (NSTEMI) (25\%), and 151 out of the 200 patients had ST elevation myocardial infarction (STEMI) (75\%).

Hypertension was present in 116 (58\%), diabetes mellitus in 50 (25\%), hyperlipidemia in 139 (69.5\%), smoking in 150 (75\%) and family history of ischemic heart disease in at least one first degree relative in 106 (53\%) patients. All of the risk factors were significantly more frequent in the patient group than in the control group $(p<0.05)$.

Blood samples were obtained and genomic DNA was isolated with the High Pure PCR Template Preparation kit (Roche Diagnostics, IN, USA). The quantity and quality of DNA in each sample were estimated by UV absorbance and agarose gel electrophoresis. Genotyping for GPIba Kozak was performed by PCR based on the GPIba gene sequence. The forward primer was GAGAGAAGGACGGAGTCGAG and the reverse primer was GGTTGTGTCTTTCGGCAGG. Each reaction contained 0.1 to $0.4 \mathrm{mg}$ genomic DNA, 5 pmol of each primer, $200 \mathrm{mmol}^{-1}$ of each dNTP, $1.0 \mathrm{U}$ of Taq DNA polymerase, $1.0 \mathrm{mM} \mathrm{MgCl}$ and $2 \mathrm{ml}$ of $10 \times$ PCR buffer provided by the manufacturer (MBI Fermentas). The amplification product (457 bp) was digested with the restriction enzyme Psp5II. PCR products were separated on $2 \%$ agarose gel and visualized by ethidium bromide staining. Digestion of the amplified product from T/T homozygotes produces three bands $(125,157,175 \mathrm{bp})$, from C/C homozygotes, two bands (125 and $332 \mathrm{bp}$ ), and from heterozygotes, four bands $(125,157,175,332 \mathrm{bp})$ for GPIba Kozak (Figure 1).

\section{Statistical analysis}

All statistical tests were performed with SPSS software, version 7.5 (SPSS, Chicago, IL, USA). Continuous data are presented as mean \pm SD, and categorical data are summarized as frequencies and percentages. The statistical significance of the differences of independent samples was examined by Student's $t$ test, and non-numerical data such as gender, genotype, hypertension, diabetes mellitus, smoking, hyperlipidemia and family history were examined by the $\chi^{2}$ test. The differences were considered to be statistically significant when $p$-values were less than 0.05 . 


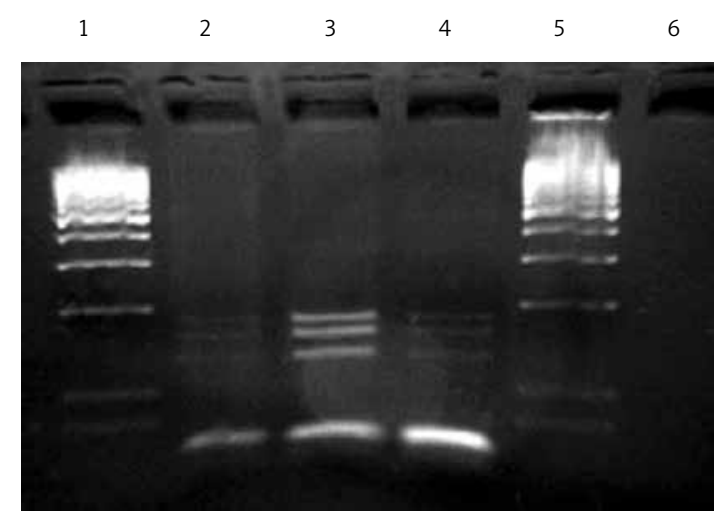

Figure 1. Assessment of GPIba Kozak polymorphism. Individuals were genotyped by cleavage of PCR products with restriction endonuclease enzyme Psp5II. 1 and 5: 100 bp DNA marker, 2-4: The enzyme cleavage products of TT genotyped individuals $(175,157,125 \mathrm{bp})$

\section{Results}

The different clinical characteristics of both ACS $(n=200)$ and control $(n=200)$ groups were analyzed with respect to GPIba Kozak genotypes, i.e. as TT and CT + CC (Table I).

In the ACS group, the mean age showed no significant difference between the TT $(n=153)$ and CT + CC groups $(n=47)(39 \pm 5$ years vs. $39 \pm 5$ years, respectively, $p=0.9)$. Similarly, in the control group regarding the mean age, no significant difference between the TT $(n=155)$ and CT + CC groups $(n=45)$ (41 \pm 5 years vs. $39 \pm 4$ years, respectively, $p=0.32$ ) was detected. There was no statistically significant difference regarding the smoking status $(p=0.7)$ between the TT (37 nonsmokers/116 smokers) and CT + CC patient groups (13 nonsmokers/34 smokers). There was no statistically significant difference regarding the gender $(p=0.24)$ between the CT + CC group (27 males/25 females) and the TT group (95 males/53 females). The family history, hypertension, hyperlipidemia and diabetes mellitus showed no significant difference between the TT group and the CT + CC group ( $p=0.86, p=0.50$, $p=0.28$ and $p=0.083$, respectively) (Table I). The different clinical characteristics of controls $(n=200)$ were also analyzed with respect to GPIb $\alpha$ Kozak genotypes, i.e. as TT and CT + CC (Table I). The different clinical characteristics of controls are shown in Table I. The age, sex, smoking status, family history, hypertension and diabetes mellitus showed no significant difference between the TT and CT + CC groups ( $p=0.32, p=0.50, p=1, p=$ $0.33, p=1, p=0.58$, respectively).

The distributions of the GPIb $\alpha$ Kozak alleles and genotypes are shown in Table II. Among the 200 ACS patients, 43 were heterozygous (CT), 4 were homozygous (CC) and 153 were homozygous (TT). The frequencies of CT, CC and TT genotypes were $21.5 \%, 2.0 \%$ and $76.5 \%$, respectively, in the ACS patients. The C allele frequency in early-onset ACS patients was $12.8 \%$, while among

Table I. Comparison of clinical characteristics of patients according to the presence or absence of GPIb $\alpha$ Kozak polymorphism in patient and control groups

\begin{tabular}{|c|c|c|c|c|}
\hline \multirow[t]{2}{*}{ Parameter } & & \multicolumn{2}{|c|}{ Genotypes } & \multirow[t]{2}{*}{$P$-value } \\
\hline & & $\begin{array}{c}C T+C C \\
N=47\end{array}$ & $\begin{array}{c}\mathrm{TT} \\
N=153\end{array}$ & \\
\hline \multirow[t]{2}{*}{ Age [years] } & ACS & $39 \pm 5$ & $39 \pm 5$ & 0.90 \\
\hline & Control & $39 \pm 4$ & $41 \pm 5$ & 0.32 \\
\hline \multirow[t]{2}{*}{ Gender (M/F) } & ACS & $27 / 25$ & $95 / 53$ & 0.24 \\
\hline & Control & $28 / 20$ & $82 / 70$ & 0.50 \\
\hline \multirow[t]{2}{*}{ Diabetes mellitus (\%) } & ACS & 15 & 28 & 0.083 \\
\hline & Control & - & 3 & 0.58 \\
\hline \multirow[t]{2}{*}{ Hypertension (\%) } & ACS & 53 & 59 & 0.50 \\
\hline & Control & 7 & 7 & 1 \\
\hline \multirow[t]{2}{*}{ Dislipidemia (\%) } & ACS & 77 & 67 & 0.28 \\
\hline & Control & 4 & 4 & 1 \\
\hline \multirow[t]{2}{*}{ Smoking (no/yes) (\%) } & ACS & $13 / 34$ & $37 / 116$ & 0.70 \\
\hline & Control & 24 & 24 & 1 \\
\hline \multirow[t]{2}{*}{ Family history (\%) } & ACS & 51 & 53 & 0.86 \\
\hline & Control & 9 & 16 & 0.33 \\
\hline
\end{tabular}

ACS - acute coronary syndrome, $C$-cytosine, $F$-female, $M$ - male, $T$ - thymidine. Statistical methods are Student's $t$-test and $\chi^{2}$ test. 
Table II. Frequency of the GPIb $\alpha$ alleles in Turkish population and its comparison with French population

\begin{tabular}{|c|c|c|c|c|c|}
\hline Locus & $\mathrm{Chr}$ & No. of alleles & Allele & \multicolumn{2}{|c|}{ Allele frequences } \\
\hline \multirow[t]{3}{*}{ Gplba Kozak } & 17 & 2 & & Turkish & French* \\
\hline & & & $\mathrm{T}$ & 0.873 & 0.868 \\
\hline & & & C & 0.127 & 0.132 \\
\hline
\end{tabular}

C-cytosine, Chr-chromosome, French - French population; No. of alleles - number of alleles, T-thymidine, Turkish - Turkish population. ${ }^{\star}$ Reference [18].

Table III. Distribution of GPIb $\alpha$ Kozak alleles and genotypes in ACS patients and controls

\begin{tabular}{|lccccc|}
\hline Variable & \multicolumn{3}{c}{ Genotypes } & \multicolumn{2}{c|}{ Alleles } \\
\cline { 2 - 6 } & TT & CT & CC & T & C \\
\hline ACS $(N=200)$ & $153(76.5 \%)$ & $43(21.5 \%)$ & $4(2.0 \%)$ & $349(87.3 \%)$ & $51(12.8 \%)$ \\
\hline Controls $(N=200)$ & $155(77.5 \%)$ & $40(20.0 \%)$ & $5(2.5 \%)$ & $350(87.5 \%)$ & $50(12.5 \%)$ \\
\hline$P$-value & & $\chi^{2}=0.233$ & $p=0.89$ & $\chi^{2}=0.011$ & $p=0.993$ \\
\hline
\end{tabular}

ACS - acute coronary syndrome, $C$ - cytosine, $T$ - thymidine. Statistical method is $\chi^{2}$ test.

the 200 control subjects, 40 were heterozygous (CT), 5 were homozygous (CC) and 155 were homozygous (TT). The frequencies of CT, CC and TT genotypes were $20 \%, 2.5 \%$ and $77.5 \%$, respectively, in the control group. The $\mathrm{C}$ allele frequency was $12.5 \%$ in the normal control group (Table III). There was no significant difference in the frequencies of the genotypes in GPIb $\alpha$ polymorphism between the early-onset ACS and control groups $\left(\chi^{2}=0.233, p=0.89\right)$. The frequency of the $C$ allele in the early-onset ACS group (12.8\%) when compared with normal controls (12.5\%) (Table III) did not differ significantly $\left(\chi^{2}=0.011, p=0.993\right)$, and $\mathrm{OR}=1.023(95 \% \mathrm{Cl}: 12.715-12.885)$.

The patients were also divided into STEMI and NSTEMI groups. In patients presenting with NSTEMI $(n=49)$, the incidence of the CT + CC genotype was of borderline statistical significance when compared to the patients presenting with STEMI $(17 / 49=35 \%$ vs. $30 / 151=20 \%$, respectively, $p=0.05)$. However, the number of patients was so small that no conclusion can be drawn.

Allele frequencies of $\mathrm{T}$ and $\mathrm{C}$ were calculated to be 0.873 and 0.128 , and no significant difference was found between Turkish and French populations (Table II).

\section{Discussion}

The main findings of this study are: (1) Frequency of Kozak polymorphism in early-onset ACS patients did not differ significantly from controls, (2) in patients presenting with NSTEMI, the incidence of the CT + CC genotype was of borderline statistical significance when compared to the patients presenting with STEMI, but the number of subjects in the two subgroups was so small that no conclusion can be drawn at this time. Further studies are then need to evaluate this finding.
Numerous platelet membrane glycoprotein polymorphisms have been identified as potential risk factors for cardiovascular disease. A nucleotide $-5 T / C$ dimorphism in the translation initiation site (Kozak sequence) of the platelet GPIba gene was associated with increased platelet surface levels of the GPIb-IX-V receptor complex [18], but there is also a publication claiming the contrary [19].

Meisel et al. [5] investigated the role of the $-5 T / C$ polymorphism of GPIb $\alpha$ as a potential risk factor for $C A D$ and adverse events complicating a coronary catheter intervention. They genotyped 1,000 patients with angiographically confirmed CAD, as well as 1,000 age- and gender-matched control subjects. Among the patients with CAD, 269 underwent percutaneous transluminal coronary angioplasty (PTCA), 103 underwent directional coronary atherectomy and 278 underwent stenting. This intervention group was followed for a 30-day composite end point of target vessel revascularization, myocardial infarction or death. Carriers of the $-5 \mathrm{C}$ allele were significantly over-represented in the group of patients developing ACS $(p=0.02)$, and the $-5 C$ allele predicted an increased risk for developing complications after PTCA. Kenny et al. [6] examined 1014 patients presenting with unstable coronary syndrome in whom 165 patients had events (recurrent ischaemia, urgent revascularization, myocardial infarction (MI), stroke and death). There was no effect of the number of $-5 \mathrm{C}$ alleles on composite endpoint frequency among Caucasian subjects, but $\mathrm{MI}$ risk increased with the number of $-5 \mathrm{C}$ alleles carried, with $\mathrm{MI}$ occurring in $2.3 \%$ of patients with the $-5 \mathrm{~T} /-5 \mathrm{~T}$ genotype, $5.0 \%$ of $-5 \mathrm{~T} /-5 \mathrm{C}$, and $16.7 \%$ of $-5 C /-5 C(p<0.01)$. Pellikka et al. studied the associations of polymorphisms of GPIba, GPIV and von Willebrand factor with the extent of coronary atherosclerosis, coronary narrowing, and 
fatal MI in an autopsy series of 300 middle-aged, Caucasian Finnish men who had suffered sudden out-of-hospital death and found that the combined ThrThr/TT haplotype of GPIba as well as the AA genotype of GPIV seem to decrease the risk of fatal MI among men during early middle age [8]. This polymorphism was found to be also associated with ischemic cerebrovascular events [9-11]. Frank et al. [12] performed genotype analysis of the Kozak sequence polymorphism of GPIb $\alpha$ in a population-based study of 18- to 44-year-old women with nonfatal MI $(n=78)$, nonfatal stroke $(n=106)$, and 384 demographically similar female control subjects. Analysis of $-5 \mathrm{~T} / \mathrm{C}$ genotypes revealed that at least one copy of the $C$ allele was present in $14.1 \%$ of $\mathrm{Ml}$ cases, $23.6 \%$ of stroke cases, and $23.7 \%$ of controls. The age-adjusted odds ratio for $\mathrm{MI}$ in women carrying at least one copy of the $C$ allele was 0.53 (95\% confidence interval (Cl): 0.27-1.05). The age-adjusted odds ratio for stroke in women carrying at least one copy of the C allele was 0.99 (95\% Cl: 0.59-1.65). Analyses stratified by stroke type (ischemic, hemorrhagic) yielded similar results. They concluded that young women carrying the $\mathrm{C}$ allele of the Kozak sequence polymorphism of GPIb $\alpha$ were not at increased risk of $\mathrm{MI}$ or stroke. They stated that, paradoxically, the $C$ allele might even be associated with a reduced risk of $\mathrm{Ml}$ in this population. Ozelo et al. [13] compared the patients $(n=180)$ presenting as survivors of $\mathrm{MI}$ with 180 controls matched by age, gender, and race. No relation was seen with the Kozak polymorphism and MI risk. Likewise, Croft et al., Candore et al. [15], and Ozelo et al. [16] reported that they could not demonstrate any relation between the Kozak polymorphism and $\mathrm{MI}$ risk. Bondarenko et al. [20] could not link Kozak polymorphism with acute ischemic stroke.

The results of this study showed that there was no significant difference between early-onset ACS patients and controls, either in genotype $\left(\chi^{2}=0.233, p=0.89\right)$ or in allele frequencies. In the present work, we found that the frequency of the $C$ allele in the early-onset ACS group when compared with normal controls did not differ significantly $\left(\chi^{2}=0.011, p=0.993\right)$ indicating that the $C$ allele is not a risk factor for early-onset ACS in the Turkish population.

The allele frequencies of GPIb $\alpha$ Kozak in the Turkish population have been estimated as shown in Table II. Allele frequencies of $\mathrm{T}$ and $\mathrm{C}$ were calculated to be 0.873 and 0.127 for the GPIba Kozak polymorphism, and no significant difference was found between Turkish and French populations.

Our paper has negative result findings from the clinical point of view. However, science is a collaborative discipline, and we think that we should report our negative results, so our colleagues do not waste their time and resources repeating our findings.

Our study has several limitations. First, we had a limited sample size, and the study population may not represent the genetic data of the whole Turkish population. Second, the absolute prevalence of each allele is so low that it appears to be of no relevance from the clinical point of view at the present time. Its clinical importance could possibly become more evident if tested in a more complex multifactorial interaction with other known and unknown factors, but this is not clear at the present time. In addition, coronary artery disease and myocardial infarction are multifactorial diseases, meaning that they are caused by a combination of genes and environment.

In conclusion, our paper has negative result findings from the clinical point of view. There was no difference of Kozak polymorphism in early-onset ACS patients compared to controls. We attribute our results to the multifactorial nature of coronary heart disease pathogenesis.

\section{Conflict of interest}

The authors declare no conflict of interest.

\section{References}

1. Napoleao P, Morteiro Mdo C, Cabral LB, et al. Changes of soluble CD40 ligand in the progression of acute myocardial infarction associate to endothelial nitric oxide synthase polymorphisms and vascular endothelial growth factor but not to platelet CD62P expression. Transl Res 2015; 166: 650-9.

2. Shen GQ, Girelli D, Li L, et al. Multi-allelic haplotype association identifies novel information different from single-SNP analysis: a new protective haplotype in the LRP8 gene is against and early-onset CAD and MI. Gene 2013; 521: 78-81.

3. Shen GQ, Girelli D, Li L, et al. A novel molecular diagnostic marker for familial and early-onset coronary artery disease and myocardial infarction in the LRP8 gene. Circ Cardiovasc Genet 2014; 7: 514-20.

4. Hartopo AB, Puspitawati I, Gharini PPR, et al. Platelet microparticle number is associated with the extent of myocardial damage in acute myocardial infarction. Arch Med Sci 2016; 12: 529-37.

5. Meisel C, Afshar-Kharghan V, Cascorbi I, et al. Role of Kozak sequence polymorphism of platelet glycoprotein Ibalpha as a risk factor for coronary artery disease and catheter interventions. J Am Coll Cardiol 2001; 38: 1023-7.

6. Kenny D, Muckian C, Fitzgerald DJ, Cannon CP, Shields DC. Platelet glycoprotein $\mathrm{lb}$ alpha receptor polymorphisms and recurrent ischaemic events in acute coronary syndrome patients. J Thromb Thrombolysis 2002; 13: 13-9.

7. Douglas H, Michaelides K, Gorog DA, et al. Platelet membrane glycoprotein Ibalpha gene -5T/C Kozak sequence polymorphism as an independent risk factor for the occurrence of coronary thrombosis. Heart 2002; 87: 70-4.

8. Pellikka M, Narhi L, Perola M, Penttila A, Karhunen PJ, Mikkelsson J. Platelet GPIbalpha, GPIV and vWF poly- 
morphisms and fatal pre-hospital $\mathrm{MI}$ among middle-aged men. J Thromb Thrombolysis 2008; 26: 91-6.

9. Esen Fl, Hancer VS, Kucukkaya RD, et al. Glycoprotein Ib-alpha Kozak polymorphism in ischemic stroke. Neurol Res 2012; 34: 68-71.

10. Song YQ, Zou HL, Zhang C. Correlation between the polymorphism of platelet glycoprotein GPIb alpha Kozak gene and transient ischemic attack. Zhonghua Yi Xue Yi Chuan Xue Za Zhi 2006; 23: 561-4.

11. Hsieh K, Funk M, Schillinger M, et al. Vienna Stroke Registry. Impact of the platelet glycoprotein Ib alpha Kozak polymorphism on the risk of ischemic cerebrovascular events: a case-control study. Blood Coagul Fibrinolysis 2004; 15: 469-73.

12. Frank MB, Reiner AP, Schwartz SM, et al. The Kozak sequence polymorphism of platelet glycoprotein Ibalpha and risk of nonfatal myocardial infarction and nonfatal stroke in young women. Blood 2001; 97: 875-9.

13. Ozelo MC, Origa AF, Aranha FJ, et al. Platelet glycoprotein Ibalpha polymorphism modulate the risk for myocardial infarction. Thromb Haemost 2004; 92: 384-6.

14. Croft SA, Hampton KK, Daly ME, Steeds RP, Channer KS, Samani NJ. Kozak sequence polymorhism in the platelet GPIbalpha gene is not associated with risk of myocardial infarction. Blood 2000; 95: 2183-4.

15. Candore G, Piazza G, Crivello A, et al. Association between platelet glycoprotein Ib-alpha and myocardial infarction: results of a pilot study performed in male and female patients from Sicily. Ann N Y Acad Sci 2006; 1089: 502-8.

16. Ozelo MC, Origa AF, Aranha FJ, et al. Platelet glycoprotein Ibalpha polymorphisms modulate the risk for myocardial infarction. Thromb Haemost 2004; 92: 384-6.

17. Thygesen K, Alpert JS, Jaffe AS, Simoons ML, Chaitman BR, White HD; the Writing Group on behalf of the Joint ESC/ACCF/AHA/WHF Task Force for the Universal Definition of Myocardial Infarction. Third universal definition of myocardial infarction. Eur Heart J 2012; 33: 2551-67.

18. Afshar-Kharghan V, Li CQ, Khoshnevis-Asl M, Lopez JA. Kozak sequence polymorphism of the glycoprotein (GP) Ibalpha gene is a major determinant of the plasma membrane levels of the platelet GPIb-IX-V complex. Blood 2000; 95: 2183-4.

19. Huang T, Sahud MA. Association of $\mathrm{C} 807 \mathrm{~T}, \mathrm{PI}(\mathrm{A})$, and -5 C/T Kozak genotypes with density of glycoprotein receptors on platelet surface. Thromb Res 2003; 112: 147-50.

20. Bondarenko EA, Shetova IM, Shamalov NA, et al. Analysis association of acute ischemic stroke and DNA markers in Russian and Moldavian populations. Genetika 2011; 47: 1393-401. 